
allemande

47-1| 2015

L'Allemagne et l'Europe | La déprise de l'Empire napoléonien en Allemagne en 1813

\title{
«Le roi appela et ils accoururent tous »: les provinces prussiennes cédées en 1807 dans la guerre de 1813-1814
}

\section{Nicola Todorov}

\section{(2) OpenEdition}

\section{Journals}

Édition électronique

URL : https://journals.openedition.org/allemagne/467

DOI : 10.4000/allemagne.467

ISSN : 2605-7913

Éditeur

Société d'études allemandes

Édition imprimée

Date de publication : 26 juin 2015

Pagination : 135-150

ISSN : 0035-0974

Référence électronique

Nicola Todorov, « «Le roi appela et ils accoururent tous » : les provinces prussiennes cédées en 1807 dans la guerre de 1813-1814 ", Revue d'Allemagne et des pays de langue allemande [En ligne], 47-1 |

2015, mis en ligne le 13 décembre 2017, consulté le 26 mai 2021. URL : http://

journals.openedition.org/allemagne/467 ; DOI : https://doi.org/10.4000/allemagne.467 


\title{
« Le roi appela et ils accoururent tous »: les provinces prussiennes cédées en 1807 dans la guerre de 1813-1814
}

\author{
- Nicola Todorov*
}

Un siècle après la bataille de Leipzig, Heinrich von Treitschke écrivit : «Au corps de Bülow revint le plaisir de reprendre possession des provinces occidentales perdues. Aussitôt arrivée la nouvelle de la bataille de Leipzig, le directeur des contributions westphalien von Motz mit son ancien uniforme prussien, et il se présenta à Mühlhausen comme conseiller provincial du roi de Prusse " ${ }^{(1)}$. Si l'argument destiné à prouver l'attachement profond de cet homme au roi de Prusse peut surprendre - en France, un comportement semblable aurait plutôt valu à Motz une place dans le dictionnaire des girouettes -, des historiens plus proches de nous, et qui ne sont guère suspects de verser dans un nationalisme exacerbé, ont suivi Treitschke dans cette appréciation de l'accueil réservé aux Prussiens. Ainsi, Ilja Mieck écrit encore dans les années 1990 que les «soldats prussiens furent reçus le plus amicalement dans les anciennes provinces occidentales de la Prusse, perdues en 1807, dans la Vieille Marche, en Frise orientale, dans le comté de Mark et dans la partie de Clèves située sur la rive droite du Rhin » ${ }^{(2)}$. On est allé jusqu'à considérer la Vieille Marche, rattachée au royaume de Westphalie, comme faisant pratiquement partie de la Prusse, tant ses habitants auraient afflué vers la Landwehr ${ }^{(3)}$.

* Chargé d'enseignement, chercheur associé au Centre d'histoire du XIXe siècle - Paris (EA 3550).

1 Heinrich von Treitschke, 1813, Leipzig, Hirzel, 1913, p. 152.

2 Ilja Мıск, "Die Integration preußischer Landesteile französischen Rechts nach 1814/15», in: Otto Büsch, Monika Neugebauer-Wölk (dir.), Preußen und die revolutionäre Herausforderung seit 1789, Berlin, de Gruyter, 1991, p. 345-364, ici p. 351.

3 Rudolf Ibekкen, Preußen 1807-1813, Staat und Volk als Idee und Wirklichkeit. Darstellung und Dokumentation (Veröffentlichungen aus den Archiven Preußischer Kulturbesitz), Cologne/Berlin, Grote, 1970 , p. 448. 
Correspondant aussi à la perception - ou à l'attente - de certains contemporains, comme l'universitaire Heinrich Steffens ${ }^{(4)}$, ces jugements reprennent l'idée que se faisaient les monarques de la fidélité inégale de leurs provinces. La Vieille Marche, par exemple, avait, pour la dynastie, un caractère symbolique, dont les contemporains étaient bien conscients. Ainsi, concluant son rapport sur l'administration de cette province, l'intendant français Chivaille, écrivit en 1808: « [...] Elle fut pour ainsi dire le berceau de la Monarchie, et la plus ancienne propriété de la maison de Brandebourg. En perdant la Vieille Marche le Roi de Prusse a perdu le plus ancien de tous ses titres, celui qui honorait le plus sa race $[. ..]{ }^{(5)}$. Et lorsque Napoléon déclara vouloir emporter l'adhésion des habitants du nouveau royaume de Westphalie par une administration exemplaire, il pensait certainement aux habitants de ces provinces prussiennes en posant à son frère Jérôme la question rhétorique suivante: "Quel peuple voudra retourner sous le gouvernement arbitraire prussien, quand il aura goûté les bienfaits d'une administration sage et libérale? " ${ }^{(6)}$

Cette tentative aurait totalement échoué. L'exploitation des ressources financières et humaines du royaume de Westphalie par son créateur aurait discrédité l'État westphalien et sa politique de réformes. Épuisés par les charges fiscales croissantes et l'impôt du sang - la conscription -, les habitants de ces provinces se seraient détournés de ce régime. Sa chute aurait au mieux laissé les habitants indifférents ${ }^{(7)}$, alors que dans certaines parties du royaume les représentants de l'État westphalien furent publiquement humiliés ${ }^{(8)}$.

La mobilisation populaire pendant les guerres dites de libération fait débat depuis longtemps. Pour les uns, ces guerres étaient des guerres traditionnelles. La propagande de guerre se serait limitée aux cercles intellectuels ${ }^{(9)}$. Pour les autres, celle-ci aurait mobilisé de larges masses de la population, militairement par un enrôlement volontaire, économiquement par leur soutien à l'effort financier, idéologiquement en diffusant ou faisant naître le sentiment national allemand, surtout en Allemagne du Nord ${ }^{(10)}$. L'étude de l'accueil réservé aux troupes de la $6^{\mathrm{e}}$ coalition par les habitants des départements orientaux du royaume de Westphalie, regroupant en grande partie d'anciens territoires

4 Heinz Heitzer, Insurrectionen zwischen Weser und Elbe: Volksbewegungen gegen die französische Fremdherrschaft im Königreich Westfalen, 1806-1813, Berlin, Rütten \& Loening, 1959, p. 252.

5 Archives Nationales (par la suite A. N.), 138AP, 143, dossier Vieille Marche, Compte rendu par l'intendant de la province, Chivaille, du 5 juin 1808, p. 18.

6 Correspondance de Napoléon, $\mathrm{n}^{\circ}$ 13361, lettre du 15 novembre 1807.

7 Armin Owzar, "Kassel 1813. Historiographische Deutungen eines militärischen Ereignisses ", in: Horst CARL, Ute Planert (dir.), Militärische Erinnerungskulturen vom 14. bis 19. Jahrhundert. Träger-Medien-Deutungskonkurrenzen, Göttingen, Vandenhoeck \& Ruprecht, 2012, p. 370, qui cite, pour le cas de Cassel, l'historien Arthur Kleinschmidt.

8 Stefan Brakensiek, "Strukturen eines antinapoleonischen Aufstands: Grebenstein 1813 ", in: Ute Planert (dir.), Krieg und Umbruch in Mitteleuropa um 1800. Erfahrungsgeschichten auf dem Weg in eine neue Zeit, Paderborn, Ferdinand Schöningh, 2009, p. 45-61, ici p. 45.

9 Michael Jeismann, Das Vaterland der Feinde. Studien zum nationalen Feindbegriff und Selbstverständnis in Deutschland und Frankreich 1792-1918, Stuttgart, Klett-Cotta, 1992, p. 95.

10 Karen Hagemann, "Francophobia and Patriotism: anti-French images and sentiments in Prussia and Northern Germany during the Anti-Napoleonic Wars ", French History, 18/4 (2004), p. 404-425, ici p. 404 et p. 424. 
prussiens, confrontés à plusieurs reprises à l'arrivée de troupes russes et prussiennes en 1813, puis leur participation à l'effort de guerre poursuivi jusqu'en 1814, permettra de mettre à l'épreuve l'idée de la loyauté à la dynastie prussienne.

\section{La propagande russe et prussienne}

\section{Soulever le peuple contre Napoléon}

L'année 1813 a vu déferler sur l'Allemagne une vague gigantesque de pamphlets, de caricatures et d'autres publications antifrançais. La question de savoir à quel point l'idéologie qu'ils véhiculaient s'est diffusée parmi les couches sociales inférieures reste cependant débattue ${ }^{(11)}$. Face à la puissance apparente de Napoléon - cela est certain - différents groupes de militaires et de politiques, inspirés par leur perception de la guerre d'Espagne ${ }^{(12)}$, ont cherché à recourir à d'autres formes de guerre, faisant massivement appel aux populations. Les stratégies destinées à provoquer un soulèvement en Allemagne ne faisaient pourtant pas l'unanimité. Des militaires russes comme le colonel Czernichev, de concert avec certains nobles allemands comme Wallmoden, avaient envisagé au moins depuis juin 1811 de soulever la population de l'Allemagne du Nord contre les Français, notamment en s'appuyant sur la noblesse de ces territoires $^{(13)}$. C'était sans doute faire appel à un groupe très restreint de la société, mais les concepteurs de ces projets avaient peut-être une idée plus réaliste des leviers du pouvoir de la noblesse foncière que les «patriotes " Gneisenau, Stein ou Arndt ne l'avaient de la guerre d'Espagne ${ }^{(14)}$. Toujours est-il que la police politique westphalienne soumit la noblesse à une surveillance policière particulière systématique, mais tardive et peu personnalisée ${ }^{(15)}$. Les nobles représentaient presque la moitié des personnes inscrites dans le registre de signalement de la police du royaume et se virent refuser, dès octobre 1812, des passeports pour leurs déplacements en Prusse ${ }^{(16)}$.

Les projets des « patriotes » prussiens misaient sur une insurrection générale en Allemagne du Nord en général et dans le royaume de Westphalie en particulier, appuyée sur des réseaux d'espionnage et de conspiration. Organisés par le baron vom Stein et, plus tard, Justus Gruner, ces réseaux sont un fait bien documenté ${ }^{(17)}$. Le mémoire que

11 Cf. la synthèse récente par Alexandra Bleyer, Auf gegen Napoleon! Mythos Volkskriege, Darmstadt, Primus, 2013.

12 Rainer Wohlfeil, Spanien und die deutsche Erhebung, 1808-1814, Wiesbaden, Fr. Steiner, 1965.

13 Rapport de Czernichev au tsar, en date du 17 juin 1811, Recueil de la Société impériale russe, vol. XXI, p. 110-125.

14 Pensons à la révision complète des idées sur l'origine et l'efficacité des guérilléros par Charles EsDAILE, Fighting Napoleon: Guerrillas, Bandits, and Adventurers in Spain, 1808-1814, New Haven (CT), Yale University Press, 2004.

15 Ce dont témoignent les tableaux des grands propriétaires du royaume: A.N., 400AP/94, octobrenovembre 1812 et Bibliothèque nationale russe de Saint-Pétersbourg (par la suite BNR), fonds 993, Archives Westphaliennes (A.W.), carton 6, 3098-3107.

16 Landeshauptarchiv Sachsen-Anhalt, Außenstelle Wernigerode (par la suite LHASA, WR), B 18 II, $\mathrm{n}^{\circ} 123 \mathrm{I}$, a, 129, circulaire de Bongars aux préfets du 16 octobre 1812, interdisant la délivrance de passeports aux nobles; BNR, fonds 993, Archives westphaliennes, carton 2, nº 262.

17 H. Heitzer, Insurrectionen (note 4), p. 217 sqq. ; Hermann Kriegl, Justus Karl Gruner und die Revolutionierung Deutschlands 1810-1814, Nuremberg, Friedrich-Alexander Universität, 1983. 
Stein, reprenant ses idées énoncées dès 1808, soumet au tsar Alexandre le 18 juin 1812 suggère tout un éventail de moyens pour déstabiliser le gouvernement westphalien, en inondant le territoire du royaume de tracts, de pamphlets, de fausses informations ${ }^{(18)}$. Dans ce mémoire «Sur les moyens de stimuler et maintenir la participation de l'Allemagne à la guerre contre la France ", après avoir décrit la population allemande comme mécontente de la domination française, Stein poursuit: " On peut renforcer et accroître cette disposition des esprits en Allemagne, si on y répand des écrits, qui présentent un tableau pertinent de la situation malheureuse et humiliante de ce pays ${ }^{(19)}$. Le président de la police de la ville de Berlin, Justus Gruner, avait tissé un vaste réseau d'espionnage, se concentrant particulièrement sur le royaume de Westphalie. Démantelé partiellement en 1812, ce réseau fut facilement utilisé par Gruner, exilé à Prague et doté d'importants fonds russes, depuis l'été 1812, à des fins de diversion.

En Prusse, les pasteurs luthériens furent systématiquement sollicités pour soutenir idéologiquement l'effort de guerre contre la France, comme Gneisenau l'avait souhaité dès 1808 dans son projet de provoquer une insurrection générale en Allemagne du Nord. Les pasteurs allaient d'ailleurs concourir volontairement à ce que Karen Hagemann a pu appeler une "guerre sainte ${ }^{(20)}$. Dans les provinces prussiennes intégrées au royaume de Westphalie, certains prédicateurs suivaient clairement le programme esquissé par Stein, intégrant dans leurs sermons des appels cachés à la révolte contre la domination française et à l'union des Allemands, après avoir dépeint la situation du peuple allemand comme sinistre. Ils attiraient l'attention de la police politique westphalienne, comme le pasteur Bluhdorn de la cathédrale de Magdebourg ${ }^{(21)}$. L'idée de la gallophobie de nombreux pasteurs est corroborée par l'analyse du lexique d'origine française de leurs écrits au sujet des cantonnements militaires. Ils sont nombreux, en Westphalie comme en Prusse, à avoir pratiqué un purisme linguistique allemand, écartant consciemment des termes français ${ }^{(22)}$, alors que même des auteurs antifrançais eurent du mal à s'affranchir du vocabulaire d'origine française (fig. 1).

L'apparition d'annonces publicitaires dans les journaux du royaume de Westphalie pour des dictionnaires germano-russes, ornés parfois de caricatures antifrançaises, au mois de janvier $1813^{(23)}$, un mois avant que des unités russes franchissent l'Elbe, faisait

18 Karl Freiherr vom Stein, eine Biographie, Hambourg, Verlag der modernen Klassiker, 1863, p. 258-259.

19 Ibid.

20 Karen Hagemann, «A Valorous Nation in a Holy War: War Mobilization, Religion and Political Culture in Prussia, 1807 to 1815 », in: Michael Broers, Peter Hicks, Agustin Guímérá (dir.), The Napoleonic Empire and the New European Political Culture, Basingstoke, Palgrave Macmillan, 2012, p. 186-198.

21 Nicola Todorov, "Ablösung der 'preußischen Willkürherrschaft' durch eine 'weise und liberale Verwaltung'? Die Magdeburger und der westfälische Staat », Parthenopolis. Jahrbuch für Kultur und Stadtgeschichte Magdeburgs, vol. 1, 2007/2008, Magdebourg, Mauritius, 2008, p. 103-127, ici p. 121.

22 Nicola Todorov, «Le langage comme indicateur du brassage culturel à l'époque du Premier Empire: l'exemple de l'Allemagne ", in: François Antoine, Jean-Pierre Jessenne, Annie Jourdan, Hervé Leuwers, L'Empire napoléonien - une expérience européenne?, Paris, Armand Colin, 2014, p. 423438, ici p. 432-434.

23 Rien ne démontre le caractère endogène de ces annonces, contrairement à ce que croit Claudie PAYE, "Der französischen Sprache mächtig". Kommunikation im Spannungsfeld von Sprachen und Kulturen im Königreich Westphalen 1807-1813, Munich, Oldenbourg, 2013, p. 198-206. 
Figure 1. La proportion et la diversité lexicale des mots d'origine française dans les écrits contemporains et postérieurs

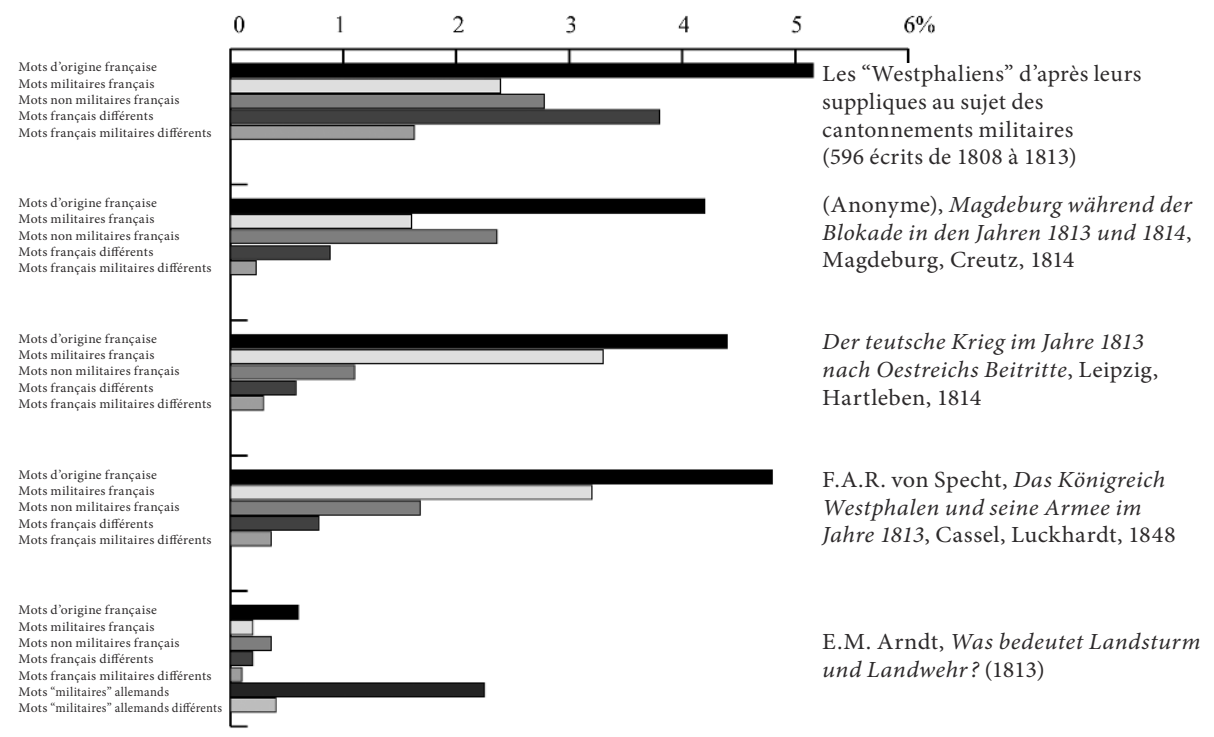

La langue allemande avait emprunté beaucoup de mots au français, notamment dans le domaine militaire. Même les auteurs d'écrits antifrançais de 1814 et postérieurs ont du mal à s'en affranchir. Mais on remarque la très relative rareté de mots français dans le pamphlet d'Arndt, qui n'a rien d'un traité militaire sur la Landwehr. Les mots " militaires » allemands qu'il utilise relèvent du champ très général du combat (« Kampf » et «Sieg»).

La correspondance westphalienne au sujet des cantonnements militaires se trouve aux archives de la Saxe-Anhalt LHASA, WR, B 18, I, 971, vol. 1-3 et B 18, II, nº 69 I a - III z (Elbe); B 26, 58, $\mathrm{n}^{\circ} 12$ vol. 1-7, B 26, 48, n 12 (Saale).

sans doute partie de cette guerre de propagande, contournant habilement la censure westphalienne. La publication d'annonces publicitaires pour faire passer des messages codés à des fins insurrectionnelles était aussi une pratique des agents de Napoléon en terre étrangère ${ }^{(24)}$. Utilisées par l'armée russe, ces annonces, après le passage des débris de la Grande Armée, ne laissaient guère de doute aux Westphaliens sur la tournure qu'avait prise la guerre. L'armée russe disposait d'un service spécial d'impression de tracts $^{(25)}$ et bénéficiait du réseau de distribution de Gruner, dans lequel les libraires occupaient une place privilégiée ${ }^{(26)}$. Pour les quelques libraires et auteurs connus, les relations avec l'armée russe et l'engagement antifrançais précoce sont établis ${ }^{(27)}$.

24 A.N./AF/IV/1674/plaquette 5/544-561, rapport de l'espion français Lawless adressé le 23 novembre 1811 à Napoléon; l'original se trouve aux Archives du ministère des Affaires étrangères (Correspondance politique, Angleterre supp.15, 1788-1811).

25 Сергейи Искюль, « Русские Листовки в Германии в 1813 году », Ежегодник германской истории, 1978, p. 302 sq.

26 H. KRIEGL, Justus Gruner (note 17), p. 193.

27 C. PAYE, Kommunikation (note 23), pour le libraire Dreyssig, p. 199 sq., pour Geissler, p. 206 sq. 
Les colporteurs aussi furent sciemment utilisés par Gruner et Stein pour diffuser de fausses informations en Westphalie, où ils firent très tôt, pendant l'été 1811, puis en 1812, notamment ceux venus de l'étranger, l'objet de mesures policières répressives, voire discriminatoires ${ }^{(28)}$. La réception réelle de cette propagande par les Westphaliens n'est, en revanche, guère documentée ${ }^{(29)}$.

\section{L'écho de la propagande d'après la police westphalienne}

Dans les sources westphaliennes de 1812 et surtout de 1813, on trouve fréquemment l'écho de ces tentatives prussiennes et russes de déstabilisation que la haute police westphalienne, la police politique, cherchait à déjouer. Mettre en parallèle les réflexions des patriotes prussiens et des Russes d'un côté et les inquiétudes de la police napoléonienne de l'autre permet de voir que cette dernière était tout sauf paranoïaque. Dès janvier 1813, la police westphalienne s'inquiétait des activités d'agents étrangers, notamment dans le département du Harz, demandant aux autorités locales de rendre compte de l'esprit public et d'arrêter les colporteurs ${ }^{(30)}$. Les rapports de plus d'une trentaine de maires du département du Harz de février 1813, répondant à une circulaire du chef de la haute police du 29 janvier, ont été conservés ${ }^{(31)}$. Négliger leurs appréciations, très majoritairement bonnes, sur leurs administrés, comme convenues et donc sans valeur, ferait oublier qu'au moins deux rapports reprochent aux habitants un esprit mauvais, voire de rébellion contre les autorités. Ainsi à Treffurth, «l'opinion ne serait pas la meilleure".

Surtout, tout en assurant le gouvernement de la fidélité des habitants, ces administrateurs, profitant de l'occasion pour transmettre des revendications, renseignent dans presque un tiers des cas sur des raisons supposées du mécontentement de la population. Le maire de la ville de Mühlhausen explique ainsi la mauvaise situation économique de la ville par le choix de Nordhausen comme chef-lieu de district au détriment de Mühlhausen ${ }^{(32)}$, témoignant d'une rivalité qui remontait à la division territoriale du royaume de 1808. Dans trois cas, les maires évoquent le déclin du commerce et la perte de débouchés pour les activités du textile. Le maire de la commune rurale de

28 Helmut Berding, "Judenemanzipation im Rheinbund ", in: Eberhard WeIs, Reformen im rheinbündischen Deutschland, Munich, Oldenbourg, 1984, p. 269-286, ici 284 sq.; sur l'emploi de colporteurs par les réseaux de Stein, cf. Karl Freiherr vom Stein (note 18).

29 C. PAYe, Kommunikatio (note 23), p. 187 sq. Plusieurs centaines de ces dictionnaires furent confisquées chez les libraires. L'absence de ces éditions des bibliothèques savantes et des catalogues des foires de Francfort et Leipzig s'explique bien mieux par leur production ad hoc par l'armée russe que, comme le croit C. Paye, par une quelconque diffusion parmi les paysans, que les autorités westphaliennes craignaient davantage qu'ils n'en avaient les preuves. Les indications sur les tirages déjà écoulés ne proviennent que des libraires, interrogés par la police et soucieux de justifier leur activité par une demande réelle.

30 LHASA, WR, B 35, I, n 21, 3, lettre du général Bongars, en date du 6 avril 1813, adressée au préfet du Harz, évoquant la circulaire du 28 janvier 1813. Des rumeurs sur de prétendus pillages pratiqués par des troupes françaises, l'apparition de mendiants déguisés en cosaques, la rumeur de la fuite de Jérôme de Cassel en avril 1813 (lettre de Bongars du 19 avril 1813) répondaient bien à l'objectif de Stein « d'exciter le peuple », cf. Karl Freiherr vom Stein (note 18), p. 262. 
Bollstedt dit les habitants ravis d'être débarrassés de la soumission à l'ancienne ville impériale de Mühlhausen, mais déplore que le village doive encore y livrer l'avoine seigneuriale. Le maire de Dingelstedt mentionne le mécontentement provoqué, non pas par l'augmentation de l'impôt foncier, mais par sa réévaluation faite au moment de la révision des cadastres et rôles d'imposition entre 1810 et 1812, selon lui, moins favorable que celle des communes voisines. Loin de témoigner d'un rejet en bloc de l'État westphalien, les maires font état de revendications très différenciées, dont certaines allaient jouer un rôle.

\section{Avant le tournant: la première épreuve}

\section{D'anciens Prussiens... fidèles au roi de Westphalie}

En février 1813, la Prusse déclare la guerre à la France. Des unités russes et prussiennes franchissent l'Elbe et envahissent les territoires orientaux du royaume de Westphalie et des départements hanséatiques. Elles sont bien accueillies dans certaines régions par une partie des habitants, mais en termes d'utilité militaire, ce soutien reste en dessous de leurs attentes. L'officier russe Benkendorf parcourt au printemps 1813 les régions du nord du royaume et doit bien reconnaître l'échec de l'opération destinée à soulever toute l'Allemagne du Nord:

«[...] les partisans ne sont que la suite d'une bataille bien gagnée. Les nouvelles formations qui vont très vite dans le pays se consolideront par un succès, disparaîtront par un échec; on ne saurait donc être trop prudent avec ce noyau du patriotisme allemand [...] le désordre, la frayeur de l'ennemi vont cesser, l'ardeur des Allemands se ralentir avec la lenteur de nos mouvements » ${ }^{(33)}$.

À la fin du mois d'avril 1813, Napoléon lance sa contre-offensive, refoulant les Russes et les Prussiens sur la rive droite de l'Elbe. Le chef de la haute police du royaume de Westphalie, le général Bongars, enquête alors sur la conduite des habitants et des administrateurs durant l'occupation des parties orientales du royaume par les troupes de l'ennemi, s'appuyant sur les rapports des autorités, mais surtout son réseau d'informateurs. Dans son rapport, Bongars détaille, canton par canton, l'attitude des habitants et administrateurs en donnant une appréciation générale par canton, appuyée et illustrée par quelques cas. Évaluation sommaire de circonscriptions de 2 à 7000 habitants, elle a au moins le mérite de faire ressortir des différences régionales. Bongars atteste aux anciennes provinces prussiennes et notamment à la Vieille Marche une conduite satisfaisante, voire bonne ${ }^{(34)}$. Il fait particulièrement l'éloge du nouveau maire du bourg Arendsee ayant refusé de prêter serment au roi de Prusse, «sous peine d'être fustigé " par les Russes. Mais ce maire va encore plus loin après la conclusion de l'armistice de Plesswitz: «Le 14 juin, il fut informé que dix hussards prussiens se dirigeaient sur Arendsee. Il réunit une cinquantaine d'habitants, les plus déterminés,

33 Archives Vorontsoff, vol. 35, pièce 55, p. 124, lettre écrite le 9 avril 1813 de Hambourg, apparemment adressée au prince Vorontsoff. Cette déception a déjà été constatée par Roger DUfrAISSE, "À propos des guerres de délivrance allemandes de 1813: problèmes et faux problèmes ", Revue de l'Institut Napoléon, 148 (1987), p. 11-44, ici p. 15.

34 BNR, AW, carton 12, 6041-6050, rapport du général Bongars au roi Jérôme sur la conduite des habitants durant l'occupation temporaire par les troupes ennemies au printemps 1813. 
en cache une partie chez lui et une partie dans les maisons environnantes. Les hussards arrivèrent bientôt et demandèrent des vivres, des chevaux et de l'argent. Le maire refusa de satisfaire en observant à ces hussards qu'en vertu de l'armistice, ils devraient déjà avoir repassé l'Elbe. Ces pillards persistèrent dans leur demande en menaçant le maire Woltersdorf de l'emmener prisonnier, ce qui le détermina à appeler son monde. Les 50 habitants armés de fourches et de bâtons parurent aussitôt et mirent en fuite les hussards prussiens " ${ }^{(35)}$.

Si des Westphaliens rejoignent l'armée prussienne, une liste établie plus tard par l'administration prussienne fait état de 77 personnes, « ayant pris du service militaire au printemps avant l'armistice " (de Plesswitz) dans une dizaine de cantons westphaliens (40690 habitants) au sud de Magdebourg, permettant de calculer un taux de 1,9 engagé pour 1000 habitants $^{(36)}$, largement inférieur à celui de la Prusse $(3,6)$. Encore ne s'agit-il pas forcément de volontaires: à peine le territoire occupé, les Prussiens ordonnent la levée de la Landwehr ${ }^{(37)}$.

\section{Les provinces hanovriennes et hessoises}

Les appréciations de Bongars montrent un contraste net entre l'attitude des cantons prussiens et des cantons hanovriens rattachés seulement en 1810 au royaume de Westphalie. Le chef de la police ne fait-il que projeter dans son tableau des clichés ou idées reçues sur l'attitude des habitants des différentes provinces? Les anciens Hanovriens passaient ainsi pour être anglophiles et dans un rapport de police d'octobre 1812 on peut lire que les habitants y «soutenaient l'idée que les Anglais débarqueraient et les délivreraient [...] que si les Anglais débarquaient, une insurrection générale éclaterait ${ }^{(38)}$. Encore ne s'agit-il là peut-être que d'un écho de la propagande russe ${ }^{(39)}$.

Les rapports conservés des autorités du département du Harz sur la conduite de ses habitants pendant l'occupation d'une partie de ce département en avril 1813 donnent une idée de l'esprit rebelle hanovrien ${ }^{(40)}$. Sans les rapports des informateurs de la police, on aurait du mal à apprécier à quel point les sous-préfets embellissent le comportement des administrés de certaines localités. Ainsi, le sous-préfet de Duderstadt, Krämer, explique l'attroupement des habitants du chef-lieu au moment de l'entrée des cosaques dans la ville par la curiosité de voir des hommes d'une culture complètement étrangère et la grande affluence le jour de l'office religieux. Quelques garçons auraient fait du bruit, mais cela ne pourrait pas être désavantageusement interprété. Ce serait à peu près tout ce qui se serait passé dans le district de Duderstadt. Dans le district de Nordhausen, à Günzerode, près du chef-lieu, des habitants auraient tiré des coups de fusil et cassé des vitres par des jets de pierre à la nouvelle de l'entrée des Russes dans la ville de Nordhausen. Dans le district d'Osterode, le sous-préfet conclut que quelques habitants ne se sont pas conduits comme il fallait. À Herzberg, un petit

35 Ibid.

36 LHASA, WR, C4, B II, nº 56, 13 décembre 1813. 
détachement de hussards westphaliens, qui cherchait à se cacher chez le maire à l'arrivée de 300 cosaques, a été trahi et de ce fait capturé. À Wanfried, dans le district de Heiligenstadt, chef-lieu du département, une commission militaire est instaurée et le maire de canton arrêté par la police.

Au total, ces rapports mentionnent une dizaine de communes où des incidents, parfois seulement soupçonnés, se sont ou se seraient produits, dans un département qui en contenait 287. Bien sûr, dans la mesure où les Russes et les Prussiens ont fait plutôt des incursions sans assurer une occupation complète de ce territoire, ils n'ont pas traversé toutes les communes, dont la majorité n'a donc pas pu manifester impunément son opposition éventuelle au gouvernement westphalien. Mais un état de rébellion ouverte ou d'anarchie, tel qu'on a pu l'évoquer pour la Hesse après Leipzig ${ }^{(41)}$, ne peut guère être mis en évidence. Surtout, ces incidents sont survenus pour l'essentiel dans les parties non-prussiennes du département du Harz: les villes minières de Clausthal, Zellerfeld, Andreasberg avaient appartenu à l'électorat de Hanovre, la commune de Wanfried à l'électorat de Hesse-Cassel. Or, le cas de la Hesse, où le déclin structurel de la proto-industrie linière était associé à la conjoncture de l'époque napoléonienne ${ }^{(42)}$, ne devrait pas être généralisé à l'ensemble du royaume.

L'insurrection antinapoléonienne espérée ne se produisit donc pas. Déçus par l'attitude des Westphaliens, les coalisés ne changèrent cependant pas de stratégie, dont la mise en œuvre ultérieure ne fut rendue possible que par le changement du rapport de forces dû à l'entrée en guerre de l'Autriche.

\section{Après le tournant}

\section{L'écroulement du royaume de Westphalie?}

Sur une partie des théâtres d'opération, Napoléon avait perdu l'initiative, notamment après la défaite de Dennewitz. Une partie de la Vieille Marche est réoccupée avant Leipzig et les autorités provisoires prussiennes ordonnent immédiatement une levée d'hommes pour l'armée régulière et la Landwehr. De petites unités russes et prussiennes parcourent les parties orientales du royaume de Westphalie, évitant de s'approcher des endroits où stationnent des unités françaises ou westphaliennes. Lors de son raid sur Cassel, capitale du royaume, dans les anciens territoires hessois, le général russe Czernichev évite soigneusement de traverser longuement les anciens territoires prussiens, en passant par la Saxe, avant de se diriger brusquement vers Cassel. Après avoir pris la capitale, qui ne s'est pas rendue si facilement ${ }^{(43)}$, il proclame la dissolution du royaume de Westphalie, comptant sur l'effet psychologique de cette proclamation. Mais face à l'arrivée de renforts français, il doit quitter Cassel, retournant

41 S. BRAKENSIEK, "Strukturen» (note 8), p. 45. Comme le fait cependant remarquer cet auteur, une étude actuelle sur ce sujet fait défaut, ce qui oblige à recourir à H. HeITzER, Insurrectionen (note 4). Il n'existe donc pas d'étude quantitative et cartographique des rébellions westphaliennes, qui permettrait de les comparer avec celles d'autres territoires, comme ceux directement annexés à la France, étudiés par Aurélien Lignereux, Servir Napoléon. Policiers et gendarmes dans les départements annexés (17961814), Seyssel, Champ Vallon, 2012.

42 R. v. Friedeburg, Ländliche Gesellschaft und Obrigkeit, Göttingen, V\&R, 1997, p. 44-45.

43 Jacques-Olivier Boudon, Napoléon et la campagne de France 1814, Paris, Armand Colin, 2014, p. 24. 
dans les territoires contrôlés par les troupes de la $6^{\mathrm{e}}$ coalition, en passant par le nord du royaume. Si la ville de Brunswick, déclassée sous Jérôme Bonaparte, l'acclame, dans les autres territoires l'administration westphalienne continue à fonctionner. À quelques jours de la bataille de Leipzig, les paysans de plusieurs communes des environs de Magdebourg se rendent dans ce chef-lieu de département pour porter plainte au tribunal de district contre leur ancien seigneur foncier au sujet d'une redevance, qui, selon eux, serait abolie par la constitution du royaume de Westphalie ${ }^{(44)}$. C'était aussi reconnaître la légitimité de cet État, que Czernichev avait pourtant déclaré caduc plus d'une semaine auparavant. Le gouvernement s'inquiétait davantage des raids russes dans les territoires orientaux du royaume que de l'esprit hostile des départements hessois, non envahis ${ }^{(45)}$. Dans le département du Harz, le sous-préfet d'Osterode, Francke, envoie encore le 24 octobre - six jours après Leipzig - un rapport à Cassel, relatant les événements intervenus pendant l'occupation temporaire russe. Mais comme au printemps, ce ne sont qu'une minorité des communes, situées dans les anciens territoires hanovriens, qui voient éclater des incidents à l'encontre d'agents westphaliens ou de personnes soupçonnées d'avoir servi d'informateurs à la police ${ }^{(46)}$.

\section{L'inégale ardeur à servir le roi de Prusse}

Les autorités de transition mises en place fixent des contingents de recrues à lever par les différentes provinces. Ainsi, la Vieille Marche doit fournir 2400 hommes pour l'armée régulière et la Landwehr, pris dans la tranche d'âge de 18 à 41 ans. Le major von Hülsen, chargé d'effectuer la levée, ne réussit à rassembler que 1400 soldats. Son secrétaire explique ce résultat médiocre par une dernière levée westphalienne, qui aurait réduit le nombre potentiel d'hommes à lever, l'enrôlement de volontaires par Lützow au printemps, mais il mentionne aussi l'émigration de nombreux hommes vers le pays de Hanovre voisin se soustrayant au service pour se faire embaucher dans l'agriculture ${ }^{(47)}$. Afin de justifier ce bilan, son secrétaire, Wetterling, conclut son rapport, daté de novembre 1813, par une remarque générale:

"L'homme ordinaire de la Vieille Marche associe à une certaine dose d'indolence naturelle, de laquelle seule une forte impulsion venant de l'extérieur peut le sortir, encore presque partout l'ancienne peur servile des châtiments corporels, qui avaient lieu dans l'ancien service militaire prussien, et par conséquent il ne peut pas encore appréhender et retenir l'idée nouvelle d'un vrai combat pour la liberté [...] Nous avons essayé de lui enlever sa peur et de lui inculquer cette idée, conformément à une instruction donnée par le gouverneur civil des provinces entre l'Elbe et le Weser, Klewitz, visant à accroître le patriotisme de la Vieille Marche » ${ }^{(48)}$.

La difficulté de lever 2400 hommes, soit 2,4\% de la population de la province, résultait sans doute aussi de l'énormité du contingent demandé, si on le compare aux levées westphaliennes antérieures. Rapporté à la population du royaume dans sa totalité, le gouvernement de Jérôme aurait dû lever 48000 hommes d'un seul coup, soit davantage

44 N. Todorov, «Ablösung der 'preußischen Willkürherrschaft' » (note 21), p. 121 sq.

45 J.-O. Boudon, Napoléon (note 43), p. 25.

46 LHASA, WR, B 35, I, n² 22, rapport du 24 octobre 1813.

47 LHASA, WR, C4, AII, n 63, rapport de Wetterling à Koepken, le 21 novembre 1813.

48 Ibid. 
que la population légalement conscriptible, pour porter «l'impôt du sang " à un tel niveau. En 1811, 46123 hommes de 20 à 26 ans étaient soumis à la conscription en Westphalie et pendant les trois ans (1810-1812) moins de 32000 furent levés ${ }^{(49)}$. Même comparées à la levée westphalienne de 1813 (20000 hommes) ${ }^{(50)}$, les exigences prussiennes paraissaient exorbitantes. Mais manifestement, les sept années d'occupation française et d'administration westphalienne n'avaient pas effacé l'image négative du service militaire prussien.

Obligé de lever des recrues dans les deux cantons qu'il administre, le baron von Roht, propriétaire du domaine de Köckte, écrit le 14 octobre 1813, donc encore avant la bataille de Leipzig, aux autorités mises en place par la Prusse:

«Quant à un enrôlement entièrement volontaire aux armées de Sa Majesté le roi de Prusse, je connais ma province paternelle en tous cas si bien qu'il ne faut pas trop compter sur elle. La pression que le gouvernement précédent exerça quasi-systématiquement touchait certes toutes les classes des habitants, mais ne pesait d'une manière vraiment écrasante que sur la classe que l'on comprenait autrefois sous le nom d'exemptés » ${ }^{(51)}$,

ce qui désigne les anciens privilégiés, c'est-à-dire la noblesse à laquelle appartient d'ailleurs ce maire de canton. Il avait clairement conscience de l'augmentation inégale de la ponction fiscale, qui frappait particulièrement la noblesse et beaucoup moins les anciens contribuables.

Pourtant, il y a eu des volontaires. L'origine socio-professionnelle des 25363 volontaires de l'armée prussienne révèle certaines différences entre les unités recrutées à l'est et à l'ouest de l'Elbe, notamment en ce qui concerne la participation paysanne. Moins de $4 \%$ dans les régiments recrutés à l'est en Prusse, les paysans auraient contribué pour presque un quart aux volontaires des régiments formés dans les provinces occidentales conquises ${ }^{(52)}$, alors que les proportions sont très proches pour les artisans. Si pour la Vieille Marche la proportion de volontaires est légèrement plus élevée $(4,5 \%$ ) que la moyenne de la Prusse $(3,6 \%$ ), qui cache des disparités énormes entre les provinces, ce taux est très nettement inférieur à celui de la Marche de Brandebourg voisine (7,3\%o), à laquelle elle avait appartenu jusqu'en 1807. Au début, les volontaires avaient le choix - ou du moins le croyaient-ils - de l'unité dans laquelle ils serviraient et ceci pouvait représenter un moindre mal qu'une affectation en tant que conscrit ${ }^{(53)}$. On devrait donc rapporter le nombre de volontaires à la population soumise à la conscription.

Des disparités plus grandes se retrouvent au sein de la Vieille Marche. Le district de Stendal, limitrophe de la Prusse, ne fournit que 1,6 volontaire pour 1000 habitants, le

49 Georg Hassel, Statistisches Repertorium über das Königreich Westphalen, Brunswick, Friedrich Viehweg, 1813, p. 3.

50 Sam A. Mustafa, « Not Enough Esprit in the Corps. The Failure of the Westphalian Army, 1807-13 », http://www.sammustafa.com/honour/wp-content/uploads/2009/07/Westphalia-Article.pdf, p. 13.

51 LHASA, WR, C4, AII, n 63, 31-32, von Roht à von Koepken, le 14 octobre 1813.

52 R. IBEkKen, Preußen (note 3), p. 443-445.

53 LHASA, WR, C4, AII, $\mathrm{n}^{\circ}$ 6, 56, instruction du gouverneur militaire (Halberstadt, le 28 février 1814), rappelant que l'engagement comme chasseur volontaire ne pourrait pas être pris en compte après le délai fixé, les volontaires devant être « livrés » aux bataillons de réserve lors de la prochaine levée. Cf. R. Dufraisse, «À propos des guerres de délivrance » (note 33), p. 19. 
Figure 2

Origine des volontaires de la Vieille Marche

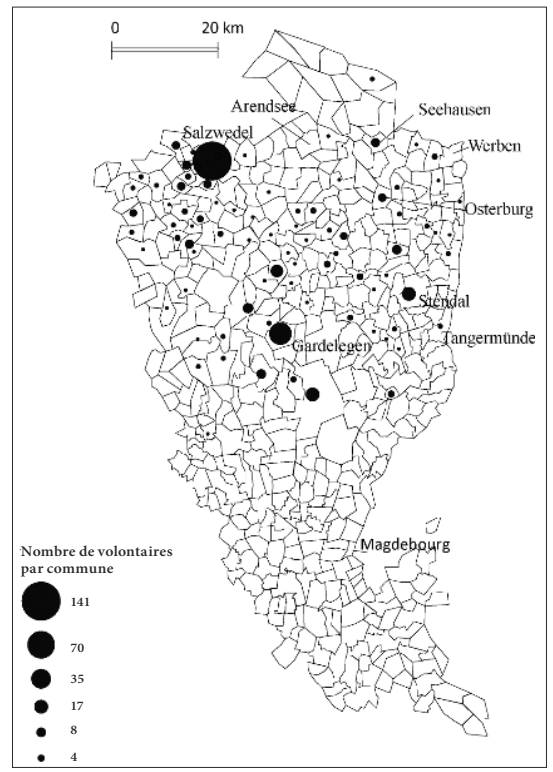

Le peuplement de la Vieille Marche

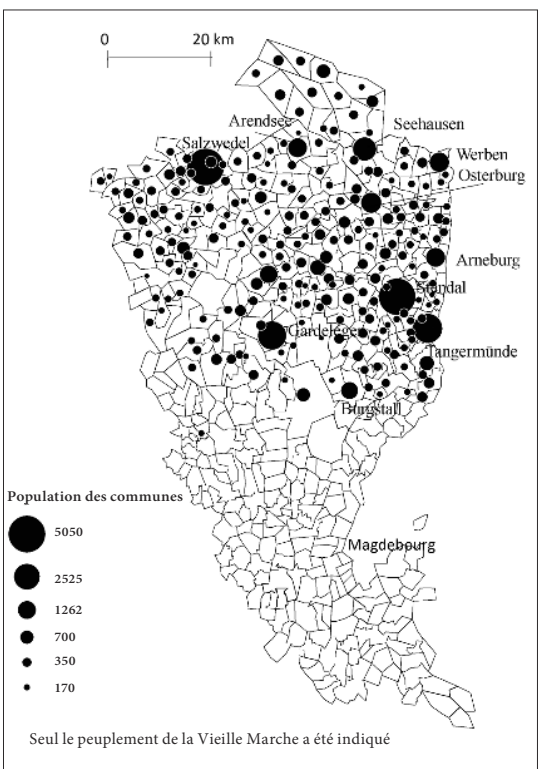

Volontaires pour l'armée prussienne dans les cantons de plusieurs districts des départements de l'Elbe et du Harz

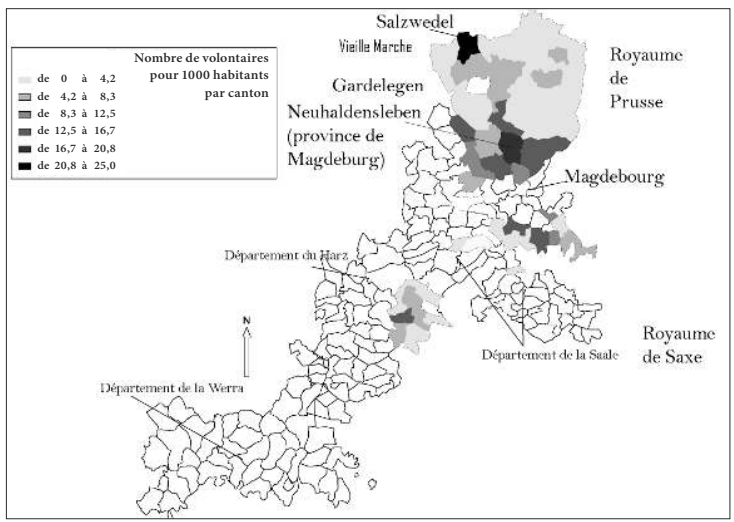

Les cartes ont été réalisées d'après les données tirées de LHASA, WR, B 18, I, 971, vol. 1-3; B 18, II, $\mathrm{n}^{\circ} 69 \mathrm{I}$ a - III z pour les incidents militaires et C 4, A, II, 61 pour les volontaires et la population. 
district de Salzwedel à l'ouest presque 8,7. Surtout, les villes de Salzwedel et de Gardelegen (19\% de la population) présentent à elles seules $53 \%$ des volontaires de ce district $^{(54)}$. Les volontaires pour les différents corps et régiments prussiens de la Vieille Marche proviennent de 84 communes dans une province qui en compte 230 (36\%). Les citadins contribuent pour $64 \%$ au volontariat, alors qu'ils ne représentent que $25 \%$ de la population (fig. 2). Le taux d'urbanisation rend compte aussi des disparités dans le recrutement des chasseurs à pied et à cheval: les deux districts de la Vieille Marche et celui de Neuhaldensleben, avec $24 \%$ de citadins, fournissent cinq fois moins de chasseurs pour 1000 habitants que l'ancien duché de Halberstadt (département de la Saale), où plus de $63 \%$ des habitants sont des citadins ${ }^{(55)}$.

Compte tenu de ce que l'on savait du profil socio-professionnel des volontaires, leur origine davantage urbaine ne surprend guère, mais elle n'explique pas les disparités entre les districts: les villes de Stendal, de Seehausen, d'Osterbourg, de Werben dans la partie orientale de la province fournissent beaucoup moins de volontaires. L'explication de ces écarts réside peut-être dans les expériences faites avec les troupes napoléoniennes entre 1807 et 1813. Les unités militaires suivaient habituellement les routes d'étape et étaient cantonnées de préférence en ville, alors que dans les villages entre deux gîtes, on ne leur servait qu'à manger et à boire. Circulant comme dans des couloirs assez étroitement balisés, les militaires ne rencontraient régulièrement qu'une minorité des habitants et avaient les contacts les plus durables avec des citadins. Les incidents entre militaires et habitants rapportés aux préfectures des départements de l'Elbe et de la Saale proviennent de $11 \%$ de leurs communes, représentant moins d'un quart de leur population $(23 \%)^{(56)}$. Or, les villes de Salzwedel et Gardelegen portent respectivement 4 et 3 affaires à la connaissance de la préfecture, alors qu'un seul incident a été rapporté par les villes de la partie orientale. S’il y avait des étapes menant au Mecklembourg dans l'est de la province, la grande route d'étapes reliant Magdebourg à Hambourg, passait par Gardelegen et Salzwedel ${ }^{(57)}$ et pourrait expliquer l'alignement des cantons présentant de nombreux volontaires. Cette répartition semble corroborer l'interprétation de K. Hagemann, selon laquelle le vécu des années de guerre expliquerait les manifestations de haine envers les Français, mais à une échelle beaucoup plus fine que son découpage sommaire entre l'Allemagne du Nord et du Sud. Surtout, cette interprétation ne pourrait guère faire abstraction de la matrice sociale déterminant l'attitude vis-à-vis des Français. Car certains bourgs ruraux et gros villages ont porté autant de plaintes au sujet des militaires à la connaissance des préfectures, sans présenter des proportions élevées de volontaires. D’ailleurs, d’une manière générale, les petites villes présentent des proportions souvent plus élevées que les chefs-lieux.

54 LHASA, WR, C4, AII, n 61, tableaux du 3 février et 14 mars 1814 (district de Stendal), du 21 février 1814 (district de Salzwedel), $1^{\text {er }}$ février 1814 (district de Neuhaldensleben).

55 LHASA, WR, C4, B II, 56. Liste des chasseurs volontaires du district de Halberstadt.

56 LHASA, WR, B 18, I, 971, vol. 1-3 et Rep. B 18 II, nº 69 I a - III z (Elbe); B 26, 58, n 12 vol. 1-7, B 26, $48, \mathrm{n}^{\circ} 12$ (Saale).

57 LHASA, WR, B 18, I, n 1020, décision du 25 juin 1810. 
Par ailleurs, les tableaux de la conscription prussienne conservés pour l'ancien département du Harz font état de proportions importantes de conscriptibles absents ${ }^{(58)}$. Sur 8486 conscriptibles de 18 à 41 ans d'une soixantaine de communes de l'Eichsfeld, 2189 sont déclarés absents, soit plus d'un quart (25,8\%), alors que le nombre de volontaires est de 205 , soit $2,4 \%$ des conscriptibles et $14 \%$ des conscrits déclarés aptes au service. Une liste de 167 conscrits réfractaires des anciens districts de Nordhausen et Heiligenstadt ${ }^{(59)}$, établie par l'administration prussienne afin de confisquer leurs biens, nous indique les professions et les propriétés des fugitifs et de leurs parents. Or, la sociologie du refus diffère quelque peu de celle du volontariat (fig. 3). Les réfractaires sont très majoritairement originaires de la campagne $(87 \%)$ et les deux tiers des familles ne disposeraient d'aucun bien.

Figure 3. Profil socioprofessionnel de 167 conscrits réfractaires prussiens des districts de Nordhausen et Heiligenstadt en 1814

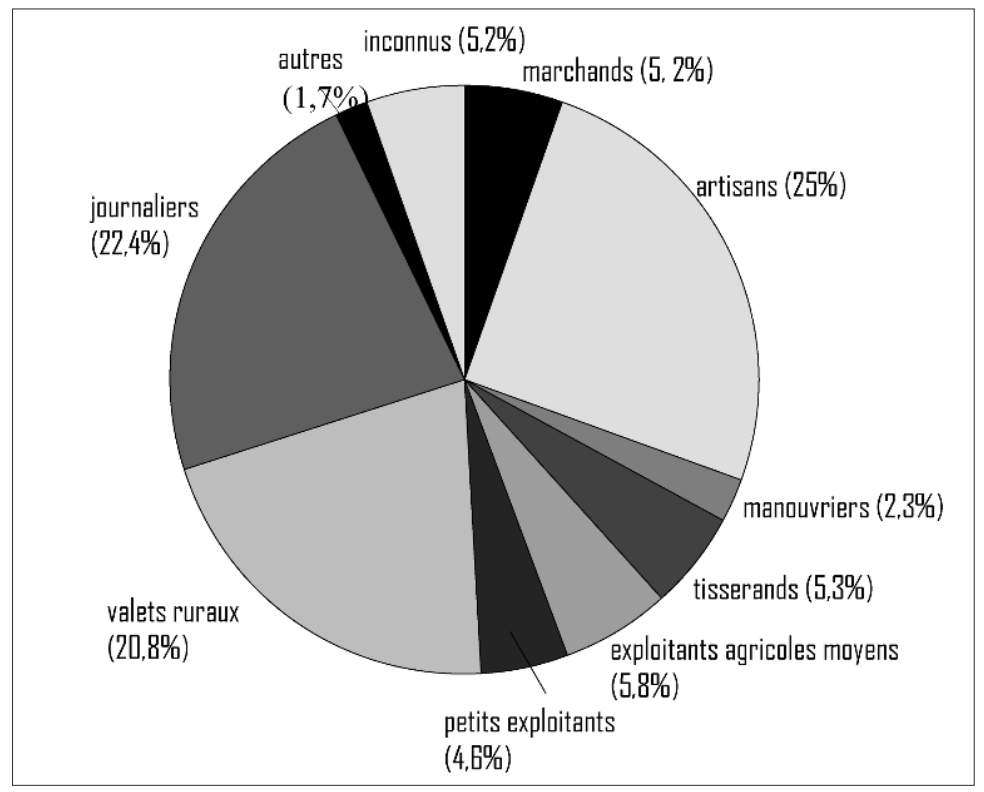

La ville de Nordhausen (moins de 9000 habitants) se distingue avec 15 conscrits réfractaires (contre 64 volontaires). À titre de comparaison, la ville de Magdebourg ( 28000 habitants) avait 27 réfractaires réfugiés en Prusse en 1811, toutes les levées westphaliennes confondues de 1808 à 1811 . Mais elle présentait 21 volontaires pour la conscription westphalienne de 1812 .

D’après une liste conservée au LHASA, WR, C5, nº 191, vol. 1, 1814.

58 LHASA, WR, B 35, 20n, n 4, liste de conscription de l'Eichsfeld et des villes de Nordhausen et Mühlhausen, 30 novembre 1813. 


\section{Conclusion}

À en croire leurs témoignages, les coalisés furent bien souvent déçus par l'attitude des anciens Prussiens, qui n'accoururent pas tous. Les attitudes face à la $6^{\mathrm{e}}$ coalition s'expliquent davantage par l'expérience des différents groupes sociaux et territoires sous la domination française que par la loyauté à l'égard des Hohenzollern. Et ces expériences étaient beaucoup plus diverses que ne l'a fait croire la propagande des coalisés. Le roi appela, oui... mais ils n’accoururent pas tous.

\section{Résumé}

Depuis Treitschke, on admettait que les anciennes provinces prussiennes cédées à Napoléon après Tilsit auraient accueilli les armées de la sixième coalition en libérateurs. Rattachées en grande partie au royaume de Westphalie, elles auraient particulièrement souffert de l'occupation militaire française et l'exploitation napoléonienne. Réexaminer cette question implique de s'intéresser à la mobilisation populaire pendant la guerre de 1813/14. Pourtant, des études qui auraient repris, à l'échelle des provinces rattachées à la Westphalie, les importants travaux de Rudolf Ibekken, menés sur le volontariat militaire de l'ensemble de la monarchie prussienne, font défaut.

Sans aucun doute, la population fit l'objet de la sollicitude des patriotes prussiens, mais leur propagande a-t-elle réellement atteint les masses populaires? L'étude des rapports de police et des préfets sur l'attitude des habitants des départements orientaux du royaume de Westphalie avant et pendant l'occupation temporaire par les troupes de la $6^{e}$ coalition, puis la participation au volontariat dans les unités formées dans ces provinces réoccupées permet de constater l'ampleur géographiquement et socialement très inégale de la mobilisation populaire. Si les raisons semblent être liées à la fois aux expériences faites durant la domination française et à des facteurs économiques et sociaux, il convient de présenter un bilan plus nuancé du royaume de Westphalie que celui diffusé par les pamphlets de la propagande prussienne, qui ont trop influencé son historiographie.

\footnotetext{
Abstract

At least since Treitschke, historians have maintained that the former Prussian provinces ceded to Napoleon after Tilsit welcomed the armies of 6 th coalition as liberators. Mainly integrated into the kingdom of Westphalia, they are thought to have suffered particularly from French occupation Napoleonic exploitation. Revisiting this question implies to focus on popular mobilization during the wars of 1813 and 1814.

However, studies taking up, at the scale of Westphalia, Rudolf Ibekken's work about voluntary military service for the whole Prussian monarchy, are lacking. Indeed, the masses were the target of propaganda, but it is much more difficult to know how far people were actually involved in this war. Police records about popular unrest and attitudes during the invasion of Westphalia's Eastern departments by Prussian and Russian troops as well as hiring of volunteers show the socially and geographically very uneven popular mobilization. The spatial patterns of this mobilization may be explained both by differences in war experience and socio-economic factors. Thus it seems necessary to give a more nuanced view of the Westphalian legacy than that left by the propaganda.
} 


\section{Zusammenfassung}

Seit Treitschke wurde angenommen, dass die 1807 an Napoleon abgetretenen preußischen Provinzen die Truppen der 6. Koalition 1813 als Befreier empfangen hätten. Vor allem die ins Königreich Westfalen integrierten Provinzen hätten besonders stark unter französischer Okkupation und napoleonischer Ausbeutung gelitten. Um diesen Sachverhalt neu zu beleuchten, ist es erforderlich, die Mobilisierung breiterer Volksmassen im Krieg von 1813 und 1814 zu untersuchen. Studien, die jedoch die Arbeiten Rudolf Ibekkens zu den Kriegsfreiwilligen für ganz Preußen im Rahmen der ins Königreich Westfalen integrierten Provinzen näher untersucht hätten, fehlen weitgehend. Breitere Volksmassen waren tatsächlich Ziel preußischer Propaganda, wieweit diese jedoch ihr Ziel erreichten, bleibt unklar. Polizeiberichte über die Haltung der Einwohner während der zweifachen Besetzung der östlichen Provinzen des Königreichs Westfalen ebenso wie die Herkunft der nach der Wiederbesetzung engagierten Kriegsfreiwilligen zeigen eher eine sozial und geografisch sehr unterschiedliche Mobilisierung der Bevölkerung. Erklärend treten die unterschiedlichen Kriegserfahrungen, soziale und ökonomische Ursachen in Erscheinung. Es scheint angebracht, ein differenzierteres Bild vom Zustand des Königreichs Westfalen zu zeichnen als jenes, das die erheblich von der preußischen Propaganda beeinflusste Geschichtsschreibung teilweise immer noch malt. 